\title{
Neutrophil to Lymphocyte Ratio and Platelet to Lymphocyte Ratio Can be Useful Markers for Distinguishing Uterine Adenomyosis and Leiomyoma
}

\author{
Yusuf MADENDAG ${ }^{1}$, Erdem SAHIN ${ }^{1}$, Emine AYDIN, Ilknur COL MADENDAG ${ }^{1}$, Gokhan ACMAZ ${ }^{1}$ \\ Hatice KARAMAN ${ }^{2}$
}

Kayseri, Turkey

\section{ABSTRACT}

OBJECTIVE: Both pelvic masses and preoperative diagnosis of them have still continued as an important investigation subject. It is important to discriminate the diagnoses of leiomyoma and adenomyosis before operation especially among infertile patients. Neoplasm can alter systemic or local immune response in their originating area. We aim to investigate using new inflammation markers, neutrophil to lymphocyte ratio and platelet to lymphocyte ratio, whether they are useful to discriminate between adenomyosis and leiomyoma.

STUDY DESIGN: We reviewed a database of all patients that had complaint from abnormal uterine bleeding resistant to medical treatment or detected pelvic mass in perimenopausal period and underwent hysterectomy in our department between January 2011 and July 2016 for study groups. However, a total of 124 patients (44 adenomyosis and 80 leiomyoma) were evaluated because of exclusion criteria and matched with 72 women who were healthy and had normal uterus and ovaries as a control group.

RESULTS: Neutrophil and platelet count, neutrophil to lymphocyte ratio and platelet to lymphocyte ratio levels were significantly higher in adenomyosis group than in control group $(p=0.039, p=0.046, p=0.001$ and $p=0.046$ respectively). Neutrophil count and neutrophil to lymphocyte ratio levels were significantly higher in adenomyosis group than in leiomyoma group ( $p=0.039$ and $p=0.001$, respectively).

CONCLUSION: As a result, high neutrophil count and neutrophil to lymphocyte ratio levels may be useful marker to discriminate between adenomyosis and leiomyoma. Therefore, the examinations, which are more invasive and more expensive such as magnetic resonance imaging, may be less need by the agency of combined use of those inflammation markers and ultrasound in preoperative period.

Keywords: Adenomyosis, Leiomyoma, Neutrophil to lymphocyte ratio, Platelet to lymphocyte ratio

Gynecol Obstet Reprod Med 2017;24(3):147-150

1 Department of Obstetrics and Gynecology, Health Sciences University Kayseri Education and Research Hospital, Kayseri

2 Department of Pathology, Health Sciences University Kayseri Education and Research Hospital, Kayseri

Address of Correspondence: Yusuf Madendag

Department of Obstetrics and Gynecology, Health Sciences University Kayseri Education and Research Hospital, 38010 Kayseri, Turkey yusufmadendag@gmail.com

Submitted for Publication:

13.06.2017

Accepted for Publication:

18.08 .2017

Access this article online

\begin{tabular}{|c|c|}
\hline \multicolumn{2}{|c|}{ Access this article online } \\
\hline $\begin{array}{c}\text { Quick Response Code: } \\
\text { Website: www.gorm.com.tr } \\
\text { info@gorm.com.tr }\end{array}$ \\
\cline { 2 - 3 }
\end{tabular}

How to cite this article: Madendag Y. Sahin E. Aydin E. Col Madendag I. Acmaz G. Karaman H. Neutrophil to Lymphocyte Ratio and Platelet to Lymphocyte Ratio Can be Useful Markers for Distinguishing Uterine Adenomyosis and Leiomyoma. Gynecol Obstet Reprod Med 2018;24(3):147150

\section{Introduction}

Both pelvic masses and preoperative diagnosis of them have still continued as an important investigation subject. It is important to discriminate the diagnoses of leiomyoma and adenomyosis before operation especially among infertile patients. Neoplasm can alter systemic or local immune response in their originating area (1). Either chronic uterine peristaltic activity or local micro- trauma caused by hyper peristaltic phase in the endometrial-myometrial interface activates the mechanism of tissue injury and repair (TIAR) (2). Infiltrative growth and chronic inflammation process follow this initial process. The platelets can be activated as a part of systemic immune response in the course of chronic inflammation (3).

Throughout TIAR process, association of macrophages and active platelets results in induction of cytokine secretion from those platelets and macrophages at the inflammation area (4). This situation, encouraging neovascularization and inflammation and the addition of endometrial cells, contributes 
to further growth of the tumor (5). Neutrophil to lymphocyte ratio (NLR) and platelet to lymphocyte ratio (PLR) can be readily determined by using of complete blood counter test (CBC). A high NLR has been shown in systemic inflammation, some gynecologic and gastrointestinal cancers and some cardiovascular diseases $(6,7)$ And also it has been reported that PLR may increase in some peripheral vascular diseases, coroner artery diseases and some gynecologic and hepatobiliary malignity and that there may be a relationship between high PLR and poor prognosis $(8,9)$.

When all these markers are considered; because of its histopathological features and being like endometriosis, adenomyosis may cause further immune response than leiomyoma, such as TIAR $(2,5)$. We aim to investigate using new inflammation markers, NLR and PLR, whether they are useful to discriminate between adenomyosis and leiomyoma. As far as we know, there is not enough data about this subject.

\section{Material Method}

This study was conducted at the Kayseri Education and Research Hospital, Department of Obstetrics and Gynecology, Kayseri, Turkey, between January 2016 and October 2016 as a retrospective cross-sectional study. Approval of the Institutional Review Board of Erciyes University was obtained in advance (2016/525).

Exclusion criteria were as fallows; diabetes mellitus, metabolic disorder, acute or chronic infection disease, hypertension, acute coroner artery disease, connective tissue disorder, vasculitis, inflammatory bowel disease, tobacco and alcohol use, renal failure, hepatitis, patients using corticosteroid drug and patients underwent blood transfusion within three months. We reviewed a database of all patients who were referred for abnormal uterine bleeding resistant to medical treatment or detected pelvic mass in postmenopausal and premenopausal period and underwent hysterectomy in our department between January 2011 and July 2016. We are capable of including 124 patients for study groups in the present study because of exclusion criteria. And also 72 healthy volunteers are evaluated for the control group.

There were three groups in our study. First group called adenomyosis was consisted of patients who were pathologically diagnosed pure adenomyosis after hysterectomy. Second group called leiomyoma was consisted of patients who were pathologically diagnosed pure leiomyoma after hysterectomy. Third group called as the control group (10) was consisted of healthy women who came to our clinic for general control and had no gynecologic neoplasia on transvaginal ultrasound and had no cancer history or gynecologic grievance (10).

In order to evaluate serum levels of hemoglobin, leukocytes, neutrophil, lymphocyte and platelet, we obtained data analysis measured preoperatively from venous blood samples of all subjects. When the neutrophil count was divided the lymphocyte count, it was named as the NLR and when the platelet count was divided the lymphocyte count, it was named as the PLR.

\section{Statistics}

To test the normality assumption of the data, Shapiro-Wilk was used. Variance homogeneity assumption was tested with Levene test. Values are expressed as mean \pm standard deviation. One-way analysis of variance (ANOVA) test was performed for the comparison of differences between groups. Tukey HSD was used for the double comparisons. All calculations were made with PASW Statistics 18 software. $\mathrm{p}<0.05$ probability value was considered as statistically significant. Additionally, age based corrected $\mathrm{p}$ values were performed.

\section{Results}

A total of 196 patients were evaluated. 44 patients with adenomyosis were matched to each 80 patients with leiomyoma or 72 patients who were healthy as a control group. The mean ages of patients in the adenomyosis group, the leiomyoma group, and the healthy control group were $48.32 \pm 5.44$ years, $47.30 \pm 4.69$ years, and $41.85 \pm 7.30$ years, respectively. The blood characteristics 196 participants of our study were represented in table 1 .

The crosscheck of age and blood parameters of all groups was represented in table 2 . In the control group age was significantly lower than in other groups. Therefore, we adjusted statistically for age and showed corrected P values in table 2.

Table 1: Characteristics of 196 the study subjects

\begin{tabular}{lc}
\hline Characteristics & Means \\
\hline Age $($ year) & $45.2 \pm 6.9$ \\
Hemoglobin $(\mathrm{g} / \mathrm{dl})$ & $12.1 \pm 1.9$ \\
Leukocyte count $(/ \mu \mathrm{L})$ & $7113 \pm 1564$ \\
Neutrophil count $(/ \mu \mathrm{L})$ & $4229 \pm 1119$ \\
Lymphocyte count $(/ \mu \mathrm{L})$ & $2236 \pm 669$ \\
Platelet count $(/ \mu \mathrm{L})$ & $291336 \pm 76662$ \\
Neutrophil to lymphocyte ratio & $1.98 \pm 0.58$ \\
Platelet to lymphocyte ratio & $138.2 \pm 45.1$ \\
Mean platelet volume $(\mathrm{fL})$ & $9.1 \pm 1.1$ \\
\hline
\end{tabular}


Table 2: The crosscheck of age and blood parameters of all groups

\begin{tabular}{|c|c|c|c|c|c|}
\hline & $\begin{array}{l}\text { Leiomyoma } \\
\qquad(n=80)\end{array}$ & $\begin{array}{c}\text { Adenomyosis } \\
(n=44)\end{array}$ & $\begin{array}{l}\text { Control patients } \\
\qquad(n=72)\end{array}$ & $\begin{array}{l}p \\
\text { Value }\end{array}$ & $\begin{array}{l}\text { Corrected } \\
p \text { Value }\end{array}$ \\
\hline Age & $47.30 \pm 4.69^{a}$ & $48.32 \pm 5.44^{a}$ & $41.85 \pm 7.30^{b}$ & $<0.001$ & \\
\hline Leukocyte & $7.08 \pm 1.57$ & $7.38 \pm 1.64$ & $6.98 \pm 1.51$ & 0.395 & 0.307 \\
\hline Hemoglobin & $11.81 \pm 2.13^{a}$ & $11.59 \pm 1.97^{a}$ & $12.85 \pm 1.62^{\mathrm{b}}$ & 0.001 & $<0.001$ \\
\hline Neutrophil & $4.18 \pm 1.05^{\mathrm{ab}}$ & $4.60 \pm 1.34^{a}$ & $4.05 \pm 1.01^{b}$ & 0.033 & 0.039 \\
\hline Lymphocyte & $2.21 \pm 0.69$ & $2.16 \pm 0.69$ & $2.32 \pm 0.63$ & 0.417 & 0.401 \\
\hline Platelet & $294.40 \pm 76.59$ & $308.27 \pm 85.66$ & $277.58 \pm 76.66$ & 0.100 & 0.046 \\
\hline Neutrophil to lymphocyte ratio & $1.99 \pm 0.95 a$ & $2.26 \pm 0.66^{b}$ & $1.80 \pm 0.46^{\mathrm{a}}$ & 0.001 & 0.001 \\
\hline Platelet to lymphocyte ratio & $141.46 \pm 44.17^{a}$ & $150.30 \pm 52.54^{a}$ & $127.44 \pm 39.29 b$ & 0.021 & 0.046 \\
\hline MPV & $9.11 \pm 1.09$ & $9.25 \pm 1.29$ & $9.19 \pm 1.18$ & 0.736 & 0.887 \\
\hline
\end{tabular}

ab:Values are expressed as mean \pm standard deviation

Neutrophil and platelet count, NLR and PLR levels were significantly higher in adenomyosis group than in control group, however we did not find a significant difference for lymphocyte count and mean platelet volume (MPV) among all groups.

Neutrophil count and NLR levels were significantly higher in adenomyosis group than in leiomyoma group, but hemoglobin, platelet count and PLR were no significantly difference between adenomyosis group and leiomyoma group. Hemoglobin count was significantly lower in both study groups than control group.

\section{Discussion}

Systemic inflammatory response is diagnosed readily by complete blood counter (CBC). NLR and PLR, which are suggested as new markers for immune response (6) can be easily calculated and are low cost markers.

In the present study, there was significantly higher mean neutrophil count in adenomyosis group than in the leiomyoma group. However, there was lower mean lymphocyte count in the study groups than in the control group, but not statistically significant. Chronic inflammation may occur owing to autoimmune diseases, malignant and benign neoplasm as well as infection. The lymphocyte count may relatively decrease and the neutrophil count increase because of chronic inflammation.

We found that mean NLR was significantly higher in the adenomyosis group than in the leiomyoma group. In many studies mean NLR values were significantly higher in malignant neoplasm groups than in benign neoplasm groups.

Either chronic uterine peristaltic activity or local microtrauma caused by hyper peristaltic phase in the endometrialmyometrial interface activates the mechanism of tissue injury and repair (TIAR) (2). The lymphocyte count may relatively decrease and the neutrophil count increase because of TIAR.

Uterine leiomyoma, which arise from the smooth muscle cells of the myometrium, are benign monoclonal tumors and include a lot of extracellular matrix (collagen, fibronectin, proteoglycan) and are encircled by a slim pseudo capsule of areolar tissue and pressed smooth muscle cells. However, adenomyosis, just like endometriosis, can be localized or diffuse and can be existing diffusely along the myometrium, or limited to separate area called an adenomyoma. Because of its histopathological features and being like endometriosis, adenomyosis may cause further immune response than leiomyoma, such as TIAR $(2,5)$.

In this study we found significantly higher mean platelet count in adenomyosis and leiomyoma group than in the control groups, however we did not find a significant difference between adenomyosis group and leiomyoma group.

Mean platelet count may increase due to chronic inflammation and/or iron deficiency anemia.

Because of inflammatory process, megakaryocytes in bone marrow may be affected and according to this platelet production and the release of large volume platelets from the bone marrow may increase. Therefore, mean platelet volume (MPV) may increase in some chronic inflammatory disease such as malignant or benign neoplasm. In some studies, mean MPV values were found significantly higher in malignant neoplasm group than in benign neoplasm group $(11,12)$.

However, we found no significant difference for mean MPV values among all the groups. In our healthy control groups some patients might have iron deficiency anemia because of their abnormal uterine bleeding, therefore mean MPV values in the healthy control group were higher than in control groups in other similar studies.

As far as we know, there is no study investigating mean PLR between adenomyosis and leiomyoma. In our study we could not find significantly difference for mean PLR between adenomyosis and leiomyoma, but in the adenomyosis group mean PLR was significantly higher than in the healthy control group. Accordingly, it does not seem to be useful for discriminating adenomyosis from leiomyoma. 
In some similar studies mean PLR was found significantly higher in malignant neoplasm group than in benign neoplasm group $(12,13)$.

Also we investigated serum hemoglobin levels among all groups and found that serum hemoglobin levels were significantly lower in both adenomyosis and leiomyoma group than in healthy control group. Uterine benign neoplasm can cause anemia; therefore, it should be treated meticulously medical or surgery.

As a result, high neutrophil count and NLR levels may be useful marker to discriminate between adenomyosis and leiomyoma. Our study was done with limited patients, but it may shed light on new researches about discrimination between adenomyosis and leiomyoma. Therefore, the examinations, which are more invasive and more expensive such as magnetic resonance imaging, may be less need by the agency of combined use of those inflammation markers and ultrasound in preoperative period.

: Source of founding: None.

Conflicts of interest: The authors report no conflicts of interest. Grant support \& amp; Financial disclosures: None

\section{References}

1. Mihalyi A, Mutinda KC, Simsa P, Dcbrock S, Mwenda JM, D'Hooghe TM. The role of immunologic and inflammatory factors in the development of endometriosis: indications for treatment strategies. Therapy 2005;(2):623-39

2. Leyendecker C, Wildt L, Mall G. The pathophysiology of endometriosis and adenomyosis: tissue injury and repair. Arch Gynecol Obstet 2009;(280):529-38.

3. Smyth SS, McEver RP, Weyrich AS, Morrell CN, Hoffman MR, Arepally GM et al. Platelet functions beyond haemostasis. J Thromb Haemost 2009;(7):1759-66

4. Scull CM, Hays WD, Fischer TH. Macrophage pro-inflammatory cytokine secretion is enhanced following interaction with autologous platelets. J Inflamm (Lotid) 2010;(7):53.
5. Harada T, Iwabe T, Tarakawa N. Role of cytokines in endometriosis. Fertil Steril 2001;(76):1-10

6. Bhat T, Teli S, Rijal J, Bhat H, Raza M, Khoueiry G, et al. Neutrophil to lymphocyte ratio and cardiovascular diseases: a review. Expert Rev Cardiovasc Ther 2013;(11): $55-9$

7. Proctor MJ, McMillan DC, Morrison DS, Fletcher CD, Horgan PG, Clarke SJ. A derived neutrophil to lymphocyte ratio predicts survival in patients with cancer. Br J Cancer 2012;(107):695-9.

8. Wang D, Yang JX, Cao DY, Wan XR, Feng FZ, Huang HF, et al. Preoperative neutrophil-lymphocyte and plateletlymphocyte ratios as independent predictors of cervical stromal involvement in surgically treated endometrioid adenocarcinoma. Onco Targets Ther 2013;(6):211-6.

9. Lin MS, Huang JX, Zhu J, Shen HZ. Elevation of platelet count in patients with colorectal cancer predicts tendency to metastases and poor prognosis. Hepato gastroenterology. 2012;59(118):1687-90.

10. Cho S, Cho H, Nam A, Kim HY, Choi YS, Park KH, et al. Neutrophil-to-lymphocyte ratio as an adjunct to CA-125 for the diagnosis of endometriosis. Fertil Steril 2008;(90): 2073-9.

11. Seçkin KD, Karslı MF, Yücel B, Yılmaz EA, Öz M, Balsak D et al. The Role of Mean Platelet Volume on Distinction of Submucosal Myomas from Other Etiologies in Increased Endometrial Thickness. Gynecol Obstet Reprod Med 2016; 22(1):32-5

12. Acmaz G, Aksoy H, Unal D, Ozyurt S, Cingillioglu B, Aksoy U, et al. Are neutrophil/ lymphocyte and platelet/ lymphocyte ratios associated with endometrial precancerous and cancerous lesions in patients with abnormal uterine bleeding? Asian Pac J Cancer Prev 2014;(15):1689-92.

13. Yildirim MA, Seckin KD, Togrul C, Baser E, Karsli MF, Gungor T, et al. Roles of Neutrophil/Lymphocyte and Platelet/Lymphocyte Ratios in the Early Diagnosis of Malignant Ovarian Masses, Asian Pac J Cancer Prev 2014;15(16):6881-5 